\title{
Precision Higgs Physics at the International Linear Collider
}

\section{Shin-ichi Kawada*†}

DESY

E-mail: shin-ichi.kawadadesy.de

The precision study of the $125 \mathrm{GeV}$ Higgs boson offers a new window into the search for new physics beyond the Standard Model. To confront the predictions of models with new interactions, the experimental program should be designed to achieve $1 \%$ precision over the full spectrum of Higgs boson couplings, with minimal model-dependence in the analysis and with tight control of systematic errors. In this contribution, we will explain how a precision Higgs program with these capabilities can be achieved at the proposed International Linear Collider. We will compare the capabilities of the ILC to those of the high-luminosity LHC and those of other $e^{+} e^{-}$Higgs factory proposals.

European Physical Society Conference on High Energy Physics - EPS-HEP2019 -

10-17 July, 2019

Ghent, Belgium

\footnotetext{
* Speaker.

${ }^{\dagger}$ On behalf of Linear Collider Collaboration (LCC) Physics Working Group
} 


\section{Introduction}

The discovery of Higgs-like boson with a mass of $\sim 125 \mathrm{GeV}$ [1,2] fulfilled one of the most important pieces of particle physics. However, there are still many open questions like the existence of dark matter which cannot be explained by the Standard Model (SM), we need new physics beyond the SM. This newly discovered boson would be a window to uncover new physics. Many new physics models predict the deviation from the SM, and the typical size of the deviation from the SM is estimated to be small, a few $\%$ to $\sim 10 \%$ [3]. To observe such a small deviation, we need very precise measurements of the Higgs boson.

The International Linear Collider (ILC) is one of the proposed future $e^{+} e^{-}$project. Its centerof-mass energy $(\sqrt{s})$ starts from $250 \mathrm{GeV}$ which is suitable for the precision Higgs measurements, but it is upgradable up to $1 \mathrm{TeV}$. The polarized beams will be used at the ILC; $80 \%$ for electrons and $30 \%$ for positrons. In this contribution, we will discuss the precision measurements of the Higgs boson at the ILC, mainly based on the Ref. [4].

\section{Higgs Measurements at the ILC}

We have evaluated the precision of Higgs measurements based on the full detector simulation of the International Large Detector (ILD) concept and/or Silicon Detector (SiD) concept [5]. In this contribution, we pick up two analyses; recoil mass measurement and Higgs self-coupling measurement. Summary of the analysis and the measurements of individual Higgs decay mode can be found in Ref. [4].

\subsection{Recoil Mass Measurement}

The key measurement of the Higgs boson at lepton colliders is the measurement of the absolute size of an inclusive cross section $\sigma_{Z h}$ by applying the recoil technique to $e^{+} e^{-} \rightarrow Z h$ process. Figure 1 left shows the Feynman diagram of $e^{+} e^{-} \rightarrow Z h$ process with the decay of $Z \rightarrow \mu^{+} \mu^{-}$. Since the initial state of $e^{+} e^{-}$collision is well-defined, the mass of the Higgs boson can be determined by only measuring muon momenta, without looking at any Higgs decay products. Figure 1 right shows the spectrum of recoil mass taken from Ref. [6]. This recoil technique is also applicable for $Z \rightarrow e^{+} e^{-}$and $Z \rightarrow q \bar{q}$ decay mode and has been analyzed [6, 7]. Assuming the twenty years running scenario of the ILC with beam polarization sharing $[8,9,10]$, the mass of the Higgs boson can be measured with a precision of $14 \mathrm{MeV}$, and $\sigma_{Z h}$ can be determined with $0.7 \%$ precision.

\subsection{Higgs Self-Coupling Measurement}

The measurement of trilinear Higgs self-coupling is quite important because this measurement allows testing the Higgs mechanism by measuring the Higgs potential directly. However, this measurement is very challenging due to its small cross section and the interference. The cross section of $e^{+} e^{-} \rightarrow$ Zhh at $\sqrt{s}=500 \mathrm{GeV}$ is only $\sim 0.2 \mathrm{fb}^{-1}$. Figure 2 shows the Feynman diagrams of Higgs pair production; several diagrams that do not involve trilinear Higgs coupling.

Full simulation studies at $\sqrt{s}=500 \mathrm{GeV}$ are performed by using $e^{+} e^{-} \rightarrow$ Zhh process with $Z \rightarrow q \bar{q} / v \bar{v} / \ell^{+} \ell^{-}$and $h h \rightarrow b \bar{b} b \bar{b} / b \bar{b} W W^{*}$ channels. With $4 \mathrm{ab}^{-1}$ statistics, a precision of $16.8 \%$ can be achieved on the cross section measurement of $e^{+} e^{-} \rightarrow \operatorname{Zhh}[11,12,13]$. Assuming the 


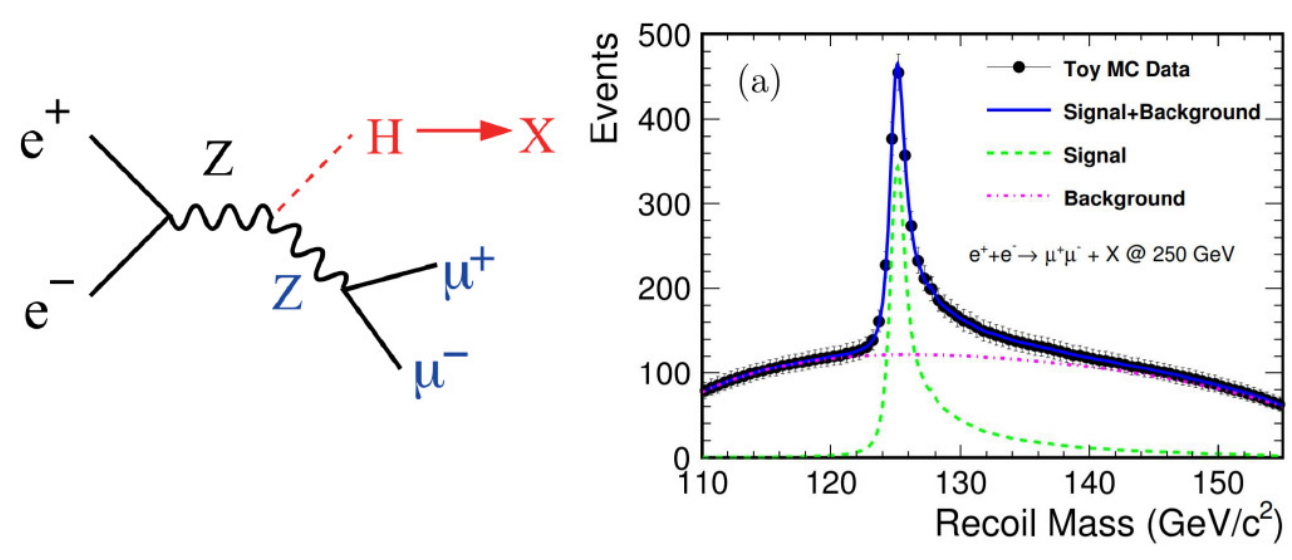

Figure 1: Left: Feynman diagram of $e^{+} e^{-} \rightarrow Z h$ with $Z \rightarrow \mu^{+} \mu^{-}$. Right: Recoil mass spectrum of events in the signal region $110-155 \mathrm{GeV}$ at $\sqrt{s}=250 \mathrm{GeV}$. Taken from Ref. [6].

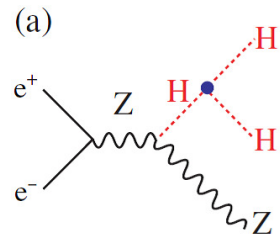

Signal diagram

(b)

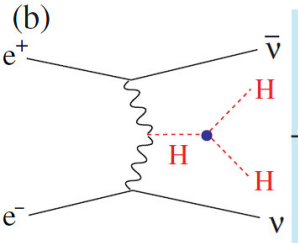

Signal diagram

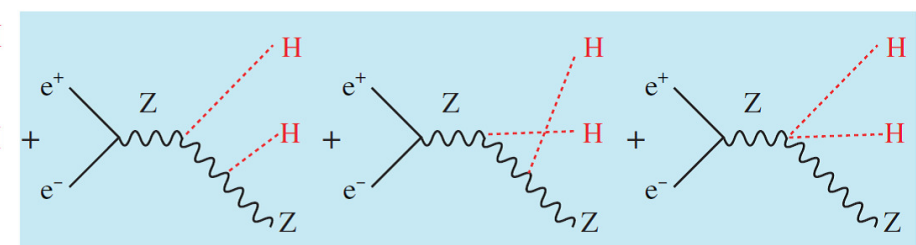

Background diagrams

Figure 2: Diagrams contributing to (a) $e^{+} e^{-} \rightarrow Z h h$ and (b) $e^{+} e^{-} \rightarrow v \bar{v} h h$.

SM with only the trilinear self-coupling free, this corresponds to an uncertainty of $27 \%$ on that coupling. At $\sqrt{s}=1 \mathrm{TeV}$, the process $e^{+} e^{-} \rightarrow v \bar{v} h h$ becomes dominant channel. With $8 \mathrm{ab}^{-1}$ data, the studies $[12,13,14]$ show that, in the same context of varying the trilinear Higgs coupling only, this coupling can be determined to $10 \%$.

Since some new physics models, in particular, electroweak baryogenesis models, predict large deviations of the trilinear Higgs coupling, it is important to see how the expected precisions would change in such cases. Figure 3 left shows the cross sections of the two reactions as a function of the triple Higgs coupling $\lambda$, and Figure 3 right shows the expected precisions at the ILC. Since the interference is different for the two reactions, constructive for $e^{+} e^{-} \rightarrow Z h h$ but destructive for $e^{+} e^{-} \rightarrow v \bar{v} h h$, these two reactions are complementary in determining trilinear Higgs coupling. If the trilinear Higgs coupling is a factor of 2 larger than SM value, the $e^{+} e^{-} \rightarrow$ Zhh process gets very useful and would provide $\sim 15 \%$ precision for the trilinear Higgs coupling. 

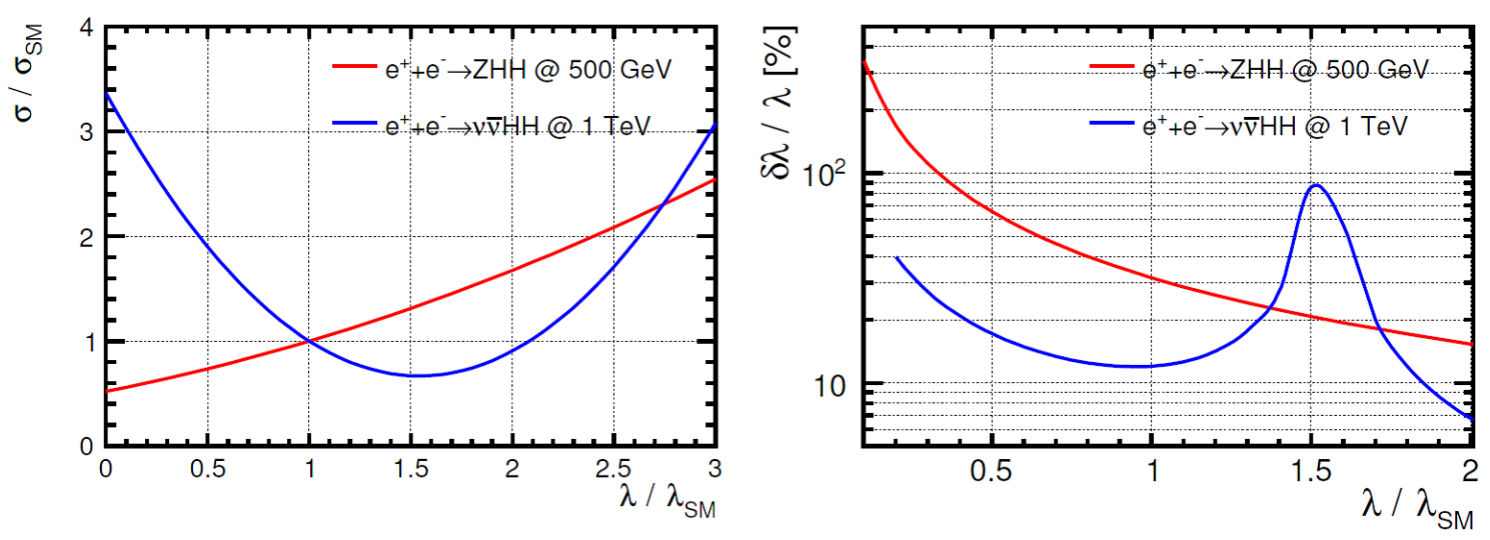

Figure 3: Left: the cross section as a function of $\lambda$ for $e^{+} e^{-} \rightarrow Z h h$ and for $e^{+} e^{-} \rightarrow v \bar{v} h h$, where values of both $\lambda$ and $\sigma$ are scaled to their SM values. Right: expected precisions of $\lambda$ when $\lambda$ deviates from its SM value. Taken from Ref. [4].

\section{Higgs Couplings - Observables to Couplings}

To extract Higgs boson couplings, we use dimension-6 SM Effective Field Theory (EFT) formalism. We use Higgs observables, triple gauge coupling observables, and electroweak precision observables as the inputs to the global fit under the EFT framework. We additionally use the ratio of branching ratio from the HL-LHC prospects as the inputs. Details of the precisions of observables and EFT framework can be found in Refs. $[4,15,16]$, here we only present the important remarks and results. The Lagrangian used in this EFT framework is Lorentz invariant, gauge invariant, and CP conserving. Though this Lagrangian has 23 free parameters, it is possible to determine all these parameters simultaneously. The Higgs couplings can be extracted in a highly model-independent way in the sense that all models of new physics describable either by the addition of local operators to the SM EFT or by the addition of invisible and exotic Higgs decays are included. In the global fitting, not only the statistical uncertainties but also systematic uncertainties have been considered [4]. Figure 4 shows the results of global fitting. Already at $\sqrt{s}=250 \mathrm{GeV}$ stage, many couplings are reached to $\sim 1 \%$ precision.

In Figure 5, a comparison has been made between polarized beams and unpolarized beams. When we compare the results of " $2 \mathrm{ab}^{-1} 250 \mathrm{GeV}$ polarized" and of " $5 \mathrm{ab}^{-1} 250 \mathrm{GeV}$ unpolarized", there are no drastic differences. In general, higher statistics always help to improve precision. However, beam polarization allows us to have more independent measurements and have better control of systematics. These facts give us more constraints in the global fitting, which is another factor of the improvement. The beam polarization is a very powerful tool, essentially compensates for a factor of 2.5 luminosity.

Finally, we compare the capabilities of the ILC for precision Higgs measurements to those of the HL-LHC, and it is shown in Figure 6. Since the assumptions for extracting Higgs couplings are different at the LHC and the ILC, we have included two additional assumptions in our EFT framework: assume no Beyond-Standard-Model decay of Higgs boson, and no anomalous couplings in $h W W$ and $h Z Z$. Even with the scenario S1, Hl-LHC plus ILC250, most of the couplings are reached $\sim 1 \%$ precision, and we can robustly claim discovery of deviations from the SM of the 


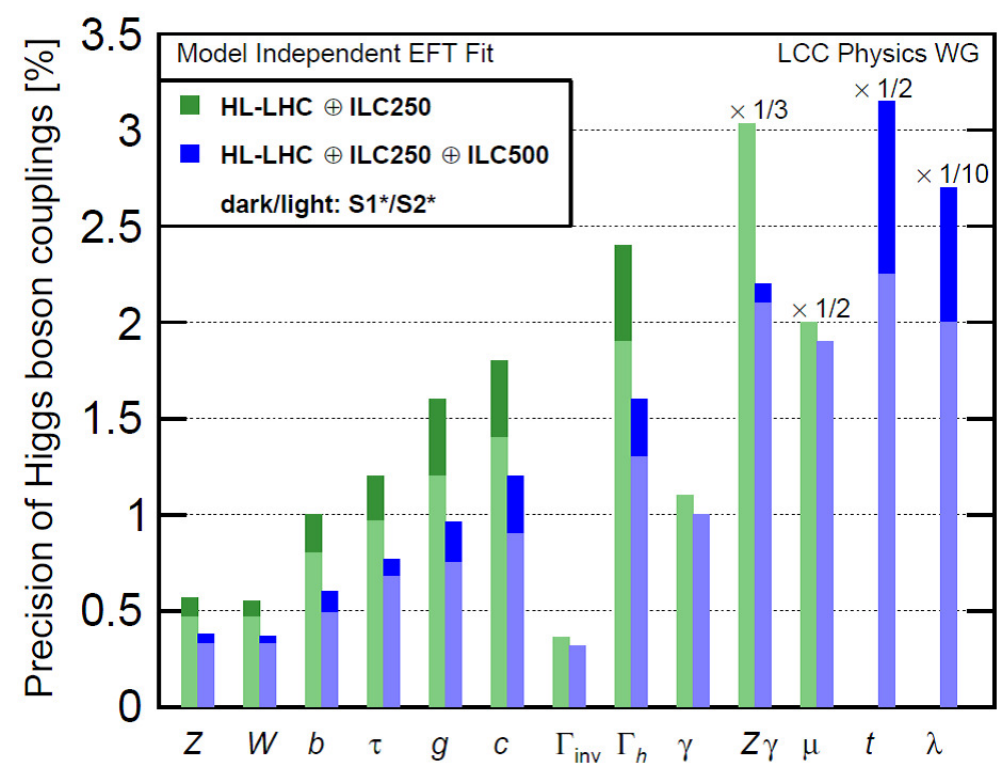

Figure 4: Projected Higgs boson coupling uncertainties for the ILC program at $250 \mathrm{GeV}$ and an energy upgrade to $500 \mathrm{GeV}$, using the highly model-independent analysis. $\mathrm{S}^{*}$ is the results based on current full simulation, and $\mathrm{S} 2^{*}$ is the expected results assuming improvements in analysis techniques and tools [4]. Taken from Ref. [4].

size generally expected in new physics models.

\section{Summary}

In this contribution, we have discussed the capabilities of measuring Higgs couplings at the ILC with two examples. The key measurement of Higgs boson at lepton colliders is the measurement of $\sigma_{Z h}$ using the recoil technique. At the ILC, the mass of Higgs boson can be measured with the precision of $14 \mathrm{MeV}$, and the $\sigma_{Z h}$ can be determined with $0.7 \%$ precision. The measurement of trilinear Higgs self-coupling is also discussed. It is possible to determine the Higgs couplings in a highly model-independent way under the EFT framework at the ILC, and most of the couplings are reached $\sim 1 \%$ precision even at $\sqrt{s}=250 \mathrm{GeV}$ stage. The beam polarization is a very powerful tool, essentially compensates a factor of $\sim 2.5$ luminosity. A comparison has been made for the prospects with HL-LHC, there are huge improvements in many Higgs couplings by adding the ILC data set.

\section{References}

[1] ATLAS Collaboration, Observation of a new particle in the search for the Standard Model Higgs boson with the ATLAS detector at the LHC, Phys. Lett. B716 (2012) 1

[2] CMS Collaboration, Observation of a new boson at a mass of $125 \mathrm{GeV}$ with the CMS experiment at the LHC, Phys. Lett. B716 (2012) 30

[3] R. S. Gupta, H. Rzehak, J. D. Wells, How well do we need to measure Higgs boson couplings?, Phys. Rev. D 86, 095001 (2012) 


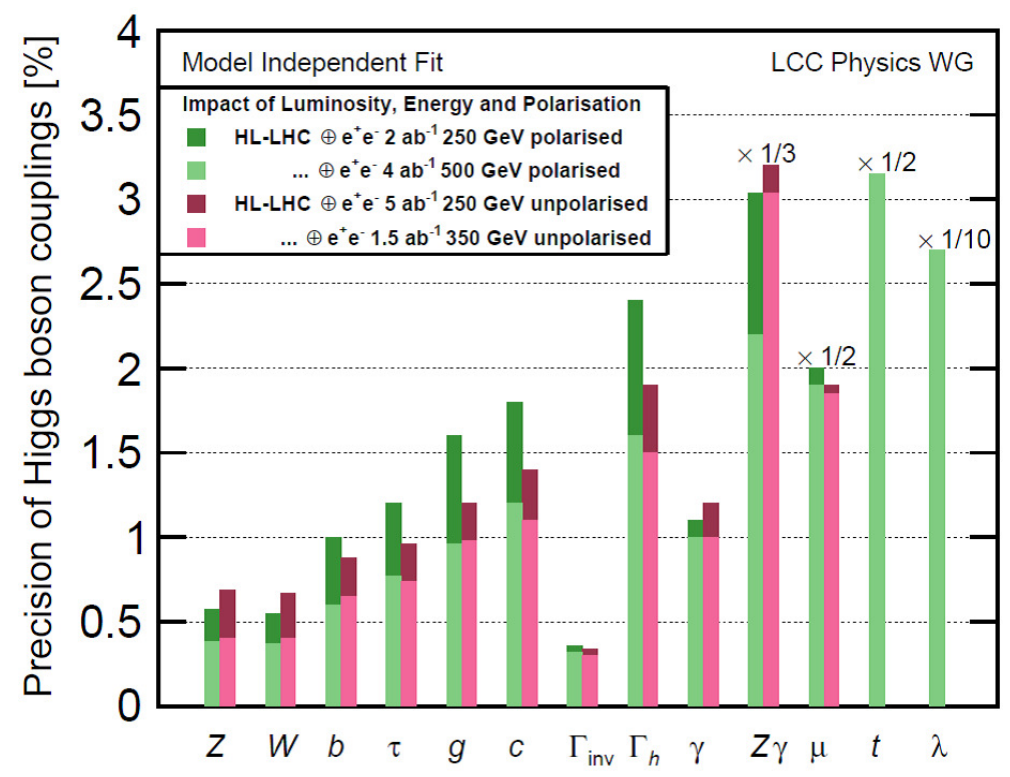

Figure 5: Projected Higgs boson coupling uncertainties for the ILC program at $250 \mathrm{GeV}$ and an energy upgrade to $500 \mathrm{GeV}$, using the highly model-independent analysis. Taken from Ref. [4].

[4] Philip Bambade et al., The International Linear Collider — A Global Project, arXiv:1903.01629 (2019)

[5] Ties Behnke et al., The International Linear Collider Technical Design Report Volume 4: Detectors, arXiv:1306:6329 (2013)

[6] J. Yan et al., Measurement of the Higgs boson mass and $e^{+} e^{-} \rightarrow Z H$ cross section using $Z \rightarrow \mu^{+} \mu^{-}$ and $Z \rightarrow e^{+} e^{-}$at the ILC, Phys. Rev. D 94, 113002 (2016)

[7] M. A. Thomson, Model-independent measurement of the $e^{+} e^{-} \rightarrow H Z$ cross section at a future $e^{+} e^{-}$ linear collider using hadronic $Z$ decays, Eur. Phys. J. C (2016) 76:72

[8] LCC Physics Working Group, Physics Case for the International Linear Collider, arXiv:1506.05992 (2015)

[9] ILC Parameters Joint Working Group, ILC Operating Scenario, arXiv:1506.07830 (2015)

[10] LCC Physics Working Group, Physics Case for the $250 \mathrm{GeV}$ Stage of the International Linear Collider, arXiv:1710.07621 (2017)

[11] Claude Fabienne Dürig, Measuring the Higgs Self-coupling at the International Linear Collider, PhD thesis, DESY-THESIS-2016-027 (2016)

[12] Junping Tian, Study of Higgs self-coupling at the ILC based on the full detector simulation at $\sqrt{s}=$ $500 \mathrm{GeV}$ and $\sqrt{s}=1 \mathrm{TeV}$, in Helmholtz Alliance Linear Collider Forum; Proceedings of the Workshops, Hamburg, Munich, Hamburg, 2010-2012, Germany (2013)

[13] Masakazu Kurata et al., The Higgs Self Coupling Analysis Using The Events Containing $H \rightarrow W W^{*}$ Decay, LC-REP-2013-025 (2013)

[14] P. Roloff et al. (CLICdp Collaboration), Double Higgs boson production and Higgs self-coupling extraction at CLIC, arXiv:1901.05897 (2019) 


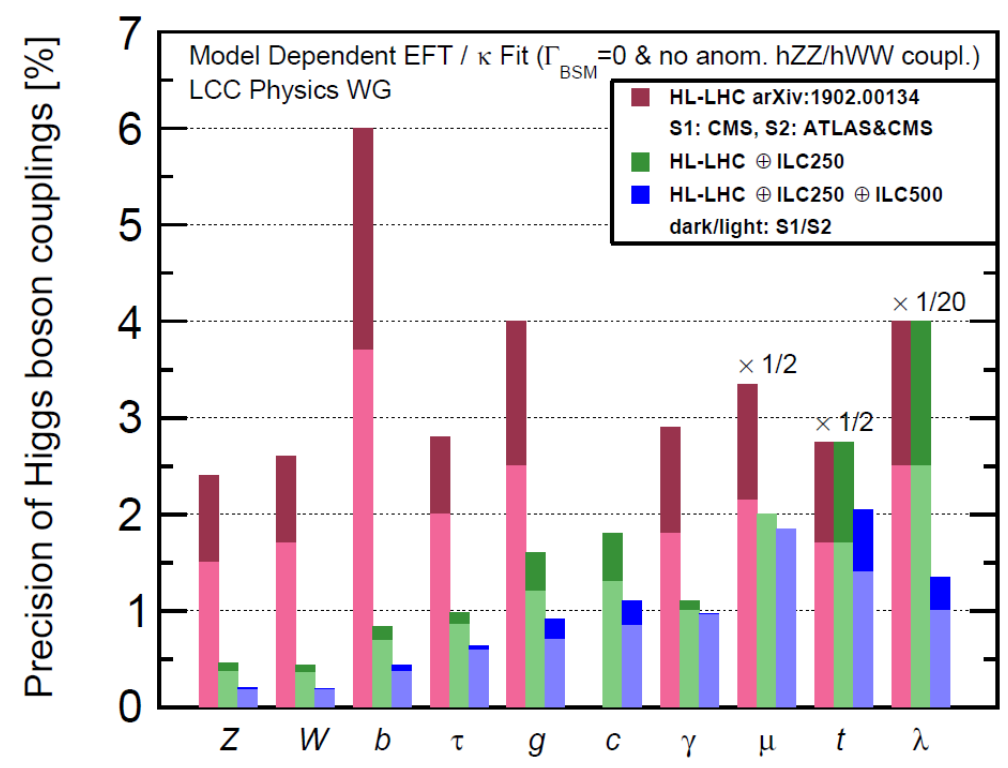

Figure 6: Projected Higgs boson coupling uncertainties for the LHC and ILC using the model-dependent assumptions appropriate to the LHC Higgs coupling fit. HL-LHC S1 is the prospects by CMS, and S2 is ATLAS and CMS. HL-LHC plus ILC S1 is the result based on current full simulation, and S2 is the expected results assuming improvements in analysis techniques and tools [4]. Taken from Ref. [4].

[15] Tim Barklow et al., Improved formalism for precision Higgs coupling fits, Phys. Rev. D 97, 053003 (2018)

[16] Tim Barklow et al., Model-independent determination of the triple Higgs coupling at $e^{+} e^{-}$colliders, Phys. Rev. D 97, 053004 (2018) 